2014

\title{
Recursive Complements and Propositional Attitudes
}

Jill de Villiers

Smith College, jdevilli@smith.edu

Kathryn Hobbs

Harvard University

Bart Hollebrandse

University of Groningen

Follow this and additional works at: https://scholarworks.smith.edu/phi_facpubs

Part of the Philosophy Commons, and the Psychology Commons

\section{Recommended Citation}

de Villiers, Jill; Hobbs, Kathryn; and Hollebrandse, Bart, "Recursive Complements and Propositional Attitudes" (2014). Philosophy: Faculty Publications, Smith College, Northampton, MA.

https://scholarworks.smith.edu/phi_facpubs/29 


\title{
Recursive Complements and Propositional Attitudes
}

\author{
Jill de Villiers, Kathryn Hobbs, and Bart Hollebrandse
}

\begin{abstract}
The focus of this chapter is in what role syntactic recursion might play in the representation of propositional attitudes. Syntactic complements under mental and communication verbs are recursive, and so also are the propositional attitudes. There is strong evidence that children take some time to master the first order syntactic complementation typical of verbs of communication. When they do acquire these structures, the evidence suggests that this helps children reason about propositional attitudes such as false beliefs. In this chapter we seek to deepen our understanding of the crucial property of sentential and attitude embedding. Is the crucial aspect that the truth value of the complement differs from that of the embedded clause, or is it that both the sentence forms and the propositional attitudes are recursive? We show that new insight can be gained by examining higher order levels of both sentence embedding and propositional attitudes. Based on some empirical data on second order complements and propositional attitude reasoning in children, we propose that truth contrasts between clauses may provide a crucial trigger for recursive complements.
\end{abstract}

Keywords Language acquisition - False beliefs - Propositional attitude • Complement $\bullet$ Speech act verb • Attitude embedding

J. de Villiers $(\bowtie)$

Department of Psychology, Smith College, Northampton, MA, USA

e-mail: jdevilli@smith.edu

K. Hobbs

Department of Psychology, Harvard University, Cambridge, MA, USA

e-mail: khobbs@fas.harvard.edu

B. Hollebrandse

Dutch Department, University of Groningen, Groningen, The Netherlands

e-mail: b.hollebrandse@ rug.nl 


\section{What Use Is Recursion?}

The focus of our interest is in what role syntactic recursion might play in the representation of propositional attitudes. Syntactic complements under mental and communication verbs are recursive, and so also are the propositional attitudes:

1) Peter thought that Kate believed that Bart said that Jill would write it

There is strong evidence that children take some time to master first order complementation, as in:

2) The girl said there was a butterfly in her hair (but it is a leaf)

What did the girl say there was in her hair?

(correct: a butterfly, wrong: a leaf))

When they do, the evidence suggests that this helps children reason about propositional attitudes like false beliefs, namely that another person has a belief that is false relative to what you believe.

In this chapter we ask what is it that matters about this first order relationship: is the crucial aspect the embedding of a different truth-value in the complement than the matrix clause, or is it that both the sentence forms and the propositional attitudes are recursive? In this chapter we review the reasoning about the relationship and its interpretation. The obvious question emerges: what about higher level orders of both sentence embedding and of propositional attitudes? We review some empirical data on second order complements and propositional attitude reasoning in children, and propose that truth contrasts between clauses may provide a crucial trigger for recursive complements.

\section{The Representational Medium}

To represent propositional attitudes in the mind requires a medium that can capture all the subtle properties of intentionality, opacity and recursion (Fodor 1975, 2008; Segal 1998; Collins 2000). Intentionality refers to the fact that mental states have contents, and these contents can be not just singular things but whole propositions. One can imagine an apple, but one can also imagine, or believe, or forget, that the apple fell behind the sideboard. So the medium in question for representation must be capable of representing propositions, that is, structured strings of symbols with a truth-value. Opacity refers to the fact that the contents of mental states may in fact be nonexistent in reality. That means one can have beliefs about nonexistent objects, such as unicorns, or angels. Crucially, the contents of mental states look inwards to mental referents, not outwards to external referents. For instance, the statement in 3):

3) Peter believes there is a ball behind the sideboard 
is not false, even though the object in question is an apple. To Peter, it is a ball. Finally, propositional attitudes are recursive, in that it is possible to have beliefs about beliefs:

4) Jill believes Peter believes there is a ball behind the sideboard

What kind of medium can these thoughts exist in? Images won't do. Although images can be recursive (e.g. fractals), and they can represent non-existent things (e.g. a unicorn), they do not have a truth value, and hence cannot be used to represent false or negative things (Fodor 1975).

Words won't do. Lexical items are not rich enough to capture propositions. If we want to say that John thought that his hat was his wife (Sacks 1985), we could say he was deluded, or mistaken, but that would not capture the content of his mistake. The contents of a propositional attitude are essential to its role in causal relations to other mental states and to behavior (Collins 2000).

A sentence, however, is just right. Several have argued that natural language sentences have the properties needed, although there is theoretical disagreement about whether the representational medium is a Language of Thought (Fodor 1975, 2008), Logical Form in natural language (Carruthers 2002) or Total Forms of natural language itself (Segal 1998; Collins 2000). But ordinary sentences have only a single proposition, and to capture the contrast between reality and the false belief of an individual, a complement clause is required if the meaning is to be conveyed in a single sentence (see Hollebrandse and Roeper 2014, this volume, for the question of whether discourse about false beliefs can be equivalently recursive).

The complements under verbs of mental state and communication have special semantic and syntactic status. For example,

\section{5) Billy thought that it was raining}

Even when the complement clause "it was raining" is false, the whole sentence is not false. This is unlike e.g. an adjunct clause, such as one headed by when under an ordinary, non-mental verb:

\section{6) Billy left when it was raining}

If the adjunct clause is false, the whole is also false. The truth shift may be an essential property that makes complement structures ideally suited for representation of false beliefs. Chomsky (2010) argues that Internal language [I-language] evolved primarily for the advantages it conferred in reasoning. Here we have a case in point. Whether or not this is true, the structures provided by I-language are ideally suited for reasoning about propositional attitudes, though of course this reasoning can go on unconsciously. Carruthers (2002) makes a related claim that reasoning could be formulated as LF, and that only when it is cloaked in phonological form do we experience it as conscious thinking (see also Jackendoff 2011). Critically for false belief reasoning, the reasoner must be able to manipulate the content of a false 
proposition not just as false but also as causally related to the intentions and goals of the holder of the propositional attitude. For example,

7) If

Billy thinks that it is raining

Then

Billy will put up his umbrella/cancel the barbecue/put on galoshes

If the person had no way to hold that representation, but instead dismissed the proposition "it was raining" as false, that person would be unable to either predict or explain Billy's behavior.

\section{Developmental Data}

Recursion is said to be a fundamental feature of human language (Hauser et al. 2002), and complements are also claimed to be universal by some linguists (Wierzbicka 1996), though this is now in contention (Everett 2008; Sauerland and Trotzke 2011). In principle, Universal Grammar? should provide recursion as a fundamental. In actuality, the forms in E-language develop over the preschool years, both in production and comprehension. This could be because not every language has recursion of every constituent type (Hollebrandse and Roeper 2014, this volume), so a child must proceed with caution. Or it may be that embedded structures exceed the performance capacities of young children.

When do children get complements on the special class of communication and mental verbs? Production data suggest that children start producing the forms between age 2 and 3 years (Bartsch and Wellman 1995). However, the sentences are often not false complements, but are more like "opinion markers", or evaluatives (see also Diessel and Tomasello 2001), and most often self-referent rather than referring to others' states of mind:

8)

*CHI: what he xxx name \# I [/] I think ?

*CHI: I going make a trailer \# I think

*CHI: I think I will use dis [: this] color

*CHI: I think you have sugar

*CHI: I think I will

In work on wh-questions, de Villiers (de Villiers 1995) had explored how children answer two-clause questions that require long distance movement:

9) What did the girl say was in her cereal $t$ ?

These questions were initially just "control" questions for our work on barrier effects on long distance movement (de Villiers et al. 1990). However, we realized 
that the children showed significant developmental growth in how they responded to these questions, too. Critically, we introduced stories in which the person said something false, because we needed to see if the trace of the wh-question was under the scope of both verbs:

10) The girl thought there was a bug in her cereal

But it was really a raisin

What did the girl say [there was $\mathrm{t}$ in her cereal]?

To get the right answer to the wh-question entails knowing that the clause is embedded, that is, that the wh-trace is embedded under the scope of BOTH verbs. In fact, children younger than about 4 years do not do this, but answer as if the trace was only connected to the second clause:

11) The girl thought there was a bug in her cereal

But it was really a raisin

What did the girl say [there was $\mathrm{t}$ in her cereal]?

Adults, 4-year-olds say "a spider"

Children below about 3.10 answer: raisin

[What] was in her cereal? = raisin

At first we thought the children's errors might be due to a recency effect, so we turned the stories around, but the same errors occurred (de Villiers 1999). Then we entertained the obvious possibility that the young children did not understand Theory of Mind yet. Perhaps they needed to pass false belief tasks before they could answer the questions correctly.

In fact, the reverse seems to be true. Children pass these tests of complementation before they can do standard Theory of Mind tasks. The contingency between the tasks has been shown for:

A) Preschool-aged typically developing children (de Villiers and Pyers 2002; de Villiers 2005a, b; Milligan et al. 2007)

B) Oral and Signing Deaf children, whether language delayed or not (de Villiers and de Villiers 2003; de Villiers 2005a, b; Schick et al. 2007)

C) Children with language delays (LI) (de Villiers et al. 2003)

D) High functioning children with autism (Tager-Flusberg and Joseph 2005)

E) Bilingual Spanish-English children (Hobbs et al. in preparation)

F) Training studies (Hale and Tager-Flusberg 2003; Lohmann and Tomasello 2003)

It is necessary to emphasize the results with deaf children in particular. We found no delay in the development of false belief understanding in children who were native learners of American Sign Language, but serious delays in children who were learning English as their first language and thus quite delayed in their grammars. These children are bright, sociable and adept at learning, so one might expect that if 
there were an alternative way to represent others' beliefs (say by imagery, or acute attention to eyegaze cues) they might spontaneously invent it, but they did not. These findings are even more dramatic in the work of Jennie Pyers on the first cohort of Nicaraguan signers, whose pidgin Sign was apparently insufficient even as young adults for representing mental state language (Pyers and Senghas 2009).

A natural conclusion is that mastery of false beliefs rests on this aspect of language: children need the structures to represent false beliefs. This conclusion has been hotly disputed by other researchers who posit a role for language other than the representational one that we defend here (Woolfe et al. 2002; Nelson 2005; Harris 2005; Ruffman et al. 2003), for example, arguing that language is the medium by which we learn about the cultural theory of mind. It is also dismissed by infancy researchers who find evidence of some sensitivity to others' belief states using eye-gaze measures long before infants have any capacity for complex sentence representation (Onishi and Baillargeon 2005; Doherty 2006; Southgate et al. 2007). For analyses of these and other objections see de Villiers (2005a, b, 2009; Perner and Ruffman 2005; Hutto 2008; Low 2010; Apperly 2011). We proceed under the assumption that for false belief tasks that entail a decision, which may or may not be the only tasks that require representation, the evidence is strong for linguistic complementation being a powerful predictor.

We have argued that sentential complements provide the structure needed to represent the false beliefs of others, because they can embed false propositions. But questions remain about what children know when they answer a question such as 9) correctly. What kind of failure is it when children answer the question with respect only to the lower clause? In recent work (de Villiers et al. 2011, 2012; de Villiers 2009) de Villiers and Roeper have argued that children may obey an idealization suggested by Chomsky (2005) as an outcome of the Strong Minimalist thesis, that Phases should be shipped off to interpretation one Phase at a time. As a consequence, children may commit a premature semantic closure of the lower clause, a closure that is suspended in adult grammar to allow long distance wh-movement in circumscribed cases like complements. Importantly, it is tensed clauses that provide the occasion for this premature interpretation, not infinitives. The presence of tense is associated with an assertion, a proposition, therefore having a truth value:

Hypothesis 1: Mastery of a sentence with a false tensed complement clause is requisite for representing false beliefs.

\section{When Is Embedding Recursion?}

Complements under mental and communication verbs are also recursive, so can be used to report second, third, nth-level propositional attitude reports. The further question arises, do children in fact recognize at the first stage that the complement is recursive? Is embedding one complement enough to signal to the child that it is 'recursive'? Roeper (2007) and Hollebrandse and Roeper (2007) argue that 
a) True recursion is infinite

b) Languages vary in which elements they allow to be recursive

c) Languages that disallow true recursion for some element may nevertheless allow one embedding. For example:

Compounds in French: only ones are two nouns, and not free but idiomatic Possessives with 's in German: only one allowed

Serial verbs in English: "come get" but not "come get eat"

The implication of their work is that being able to embed one complement does not necessarily mean that the language has recursion of complements, if the rule extends to all such cases.

If Roeper is right, then children may be conservative, that is, perhaps only evidence from two embedding complements may provide the trigger that they are recursive in English. These data are likely to be quite rare in the input. Alternatively, perhaps sentential recursion is universal, in which case the child may not be conservative and may generalize immediately. A third possibility is that recursion of complements may be universal but lexically restricted, either by type of verb or by type of complementizer, and children are conservative until the lexical properties are established. Therefore we need to ask, is recursive complementation available to English speaking children as soon as they get complements? Roeper and Hollebrandse would argue that recursion is not automatically triggered - for complements in particular - by mastery of one level of embedding.

Empirical data are thin when it comes to recursive complementation, and vanishingly rare in spontaneous speech of children and their caregivers. Several studies were designed to see when children could handle such structures in comprehension. Notice that mastery of the false complement structure can occur some months before mastery of the false belief task (de Villiers and Pyers 2002). Perhaps a more proximate cause of the change is establishing that complements are recursive. It might be that recursion is the key to really understanding and representing false beliefs at all. In other words, only when a child gets recursive complements does she have the structures for reasoning about others' false beliefs:

Hypothesis 2: Recursive complements are needed for level 1 false belief understanding

However, there is another alternative. It is of theoretical interest to ask whether there is any systematic relationship between mastery of recursive complements and mastery of recursive propositional attitudes. Roeper (2007) has proposed that the child may not in fact have recursion of either sentences OR propositional attitudes when they pass first order complements and first order false beliefs at age 4. Perhaps true recursion is later for both. One complement may be less clear in structure, but two complements may prove to the child that the form is recursive.

In addition, several theorists of Theory of Mind have criticized the standard false belief tasks as too low-level to really capture the knowledge of propositional attitude reasoning that adults have (Dennett 1987; Hutto 2008). That is, there are arguments on the other side about first order intentionality or propositional attitudes 
(Dennett 1987) being different in kind from second order and up. Essentially, first order intentionality may not require propositional-level contents, but may be reflected in the reading of a behavioral disposition (the "killjoy" hypothesis in Dennett 1987). Imagine a 17-month-old human, watching a person fail to notice a change in the hiding place of an object. When the infant then expects the character to go to the place founded on their false belief/ignorance, this has been interpreted as false belief understanding, as if the infant had the representation "Jack thinks it's in the red box" and that fed their eyegaze direction. But it could also be an expectation based on intention understanding, such as expecting Jack to continue to have the behavioral direction he had before. Developmental psychologists are hard at work to distinguish the two alternatives empirically (Dienes and Perner 2002; Baillargeon et al. 2010; Perner 2010; Low 2010). It may be that the child can understand a situation by developing some mnemonic, or behavioral expectation, based on a rule simpler than a propositional attitude ascription. Infants seem to be doing just this as long as they do not have to make a decision: if they have to act on the information, then it appears that language competence becomes entailed. In this latter case, it is very difficult to tease apart what role language plays. Language could be serving as a tool, providing a more effective mnemonic on a mental notepad. For example, language might assist in the control processes necessary to select a response (Jacques and Zelazo 2005), or it could serve as a way of keeping track of abstract relations (Loewenstein and Gentner 2005) Or, it could be the critical representational medium (de Villiers 2005a, b).

However, consider second order intentionality, as expressed in:

"Jack thinks Jill thinks it's in the red box".

At this point, simple heuristics such as following eyegaze or keeping track of orientations seem very hard to track. Propositional attitude reasoning seems the most likely alternative: one has to have a way to represent the fact that the content of Jill's thought is not in itself the content of Jack's thought, and yet Jill as the holder of the belief in question, and the content of her belief, are under the scope of Jack's thinking. There is no clear alternative to the complex hierarchical structure: we have to know which agent has which belief and not confuse them. Philosophers such as Dennett (1987) consider the breakthrough point in intentionality to be from first to second order. It is clear that human language has the rich kind of structure necessary for generating such a representation, for holding it in memory, comparing and contrasting the contents of beliefs, and so forth.

First then, it might then be argued that true understanding of both complements and false beliefs is established when each one can be shown to be recursive. In addition, recursive complements are a most suitable medium for representing recursive propositional attitudes. For that reason, Hypothesis 3 is a strong contender:

Hypothesis 3: Recursive complements are necessary for recursive (level $2+$ ) false belief reasoning. 
One last consideration is necessary before we consider the evidence. Perhaps recursion is an incidental rather than significant feature of sentences such as:

"Jack thinks Jill thinks it's in the red box".

That is, what matters to its complexity is that the final clause is false, and therefore we need to monitor who believes that. In sentences with single complements, the falsity of the embedded proposition is a significant indicator that the clause is not an adjunct but an embedded form. Adjunct clauses (e.g. because, so, when) must have "true" as their truth value:

Jill thought that because the lid was open.

Extending this idea, the real trigger of recursion of complementation is the recognition that the complement is false.

Hypothesis 4: In order to recognize genuine complement recursion, children need truth-value contrasts between clauses.

\section{Empirical Studies on Second-Order Recursion}

In our first study (Hollebrandse et al. 2006) we tested whether second-order complements (i.e. recursion in the syntax) was a predictor of false belief reasoning. We tried to divorce recursion from falsity of the complements to see what was most important, testing the various Hypotheses 1-4.

Hypothesis 1: Mastery of a sentence with a false tensed complement clause is requisite for representing false beliefs.

Hypothesis 2: Recursive complements are needed for level 1 false belief understanding

Hypothesis 3: Recursive complements are necessary for recursive (level 2+) false belief reasoning.

Hypothesis 4: In order to recognize genuine complement recursion, children need truth-value contrasts between clauses.

We devised short stories in which different people spoke about what others said, and rather than varying truth value, we varied the likely opinion of different characters, to provide some pragmatic rationale for using recursive complements. For example, Fig. 1 provides the picture for a short story:

12) Billy is talking to Jane. Mom is cleaning up Billy tells Jane that Mom said that comic books are stupid 
Fig. 1 A sample picture from the second order complements about attitudes task (Hollebrandse et al. 2008) (Figure: author's own)

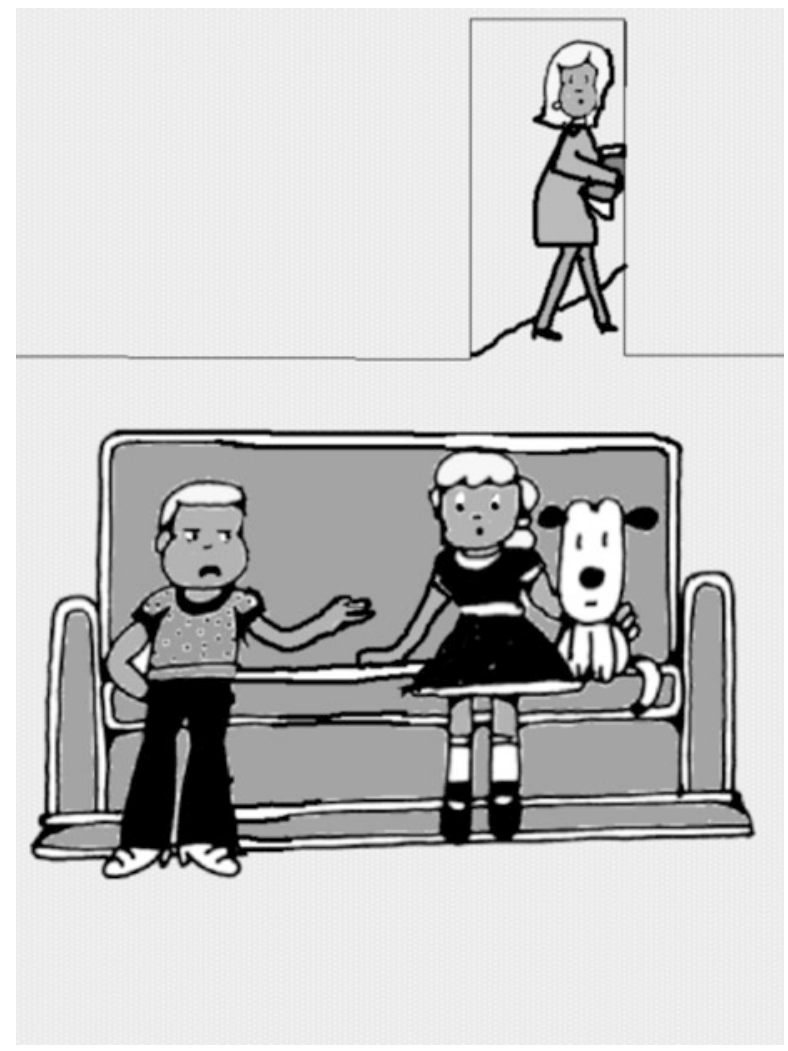

Just as in the first order complement task, we asked a wh-question to elicit an answer that would enable us to see if the child understood the embedding. We devised two different wh-questions because some adults told us that the first type of question was ambiguous:

\section{3) Type 1: What Question}

What did Billy tell Jane?

(Mom says that comic books are stupid/*comic books are stupid)

\section{4) Type 2: Who Question}

Who said that comic books are stupid?

(Mom/*Billy)

The results show that only by about 8 years of age were children successful at answering the two kinds of questions (Hollebrandse et al. 2008).

A mistake that children commonly made was to answer with first order instead of second order: 


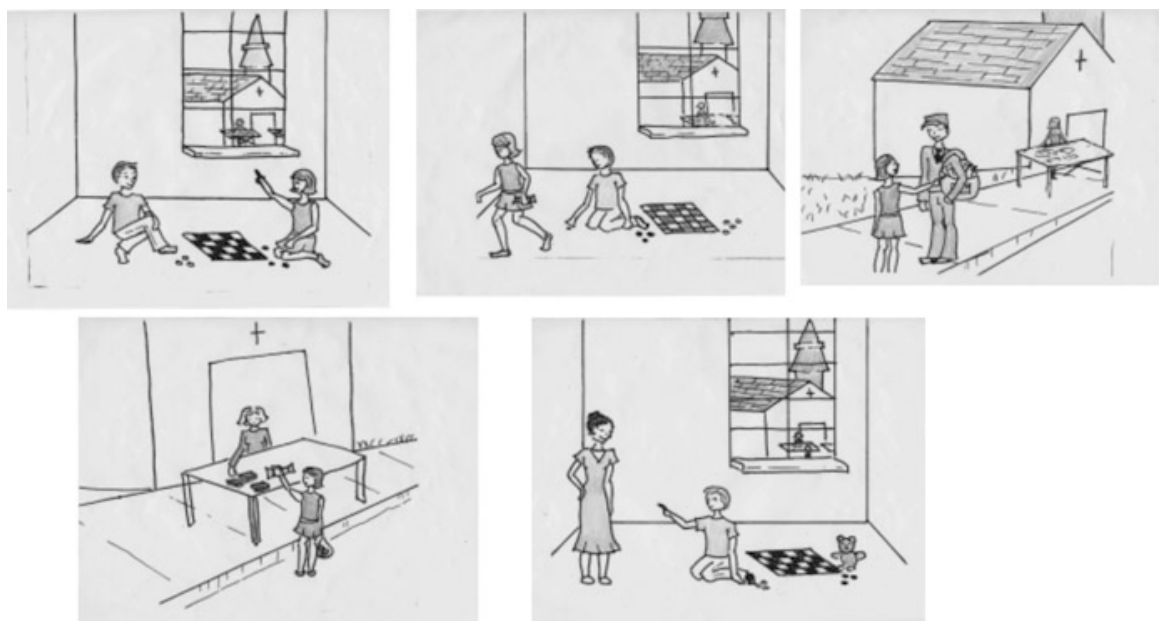

Fig. 2 The bake sale second order belief task (Hollebrandse et al. 2008) (Figure: author's own)

15) Billy tells Jane that Mom said that comic books are stupid What did Billy tell Jane?

2nd order:

"Mom says comic books are stupid"

First order:

* "Comic books are stupid"

It is very clear from this first finding that Hypothesis 2 is not supported by these facts. The children aged 5-6 years were well above the age at which first order explicit false belief tasks are passed. Therefore passing first order false belief tasks does not depend on mastery of second order, recursive complementation. Furthermore, second order complementation is difficult for children of this age even when falsehood is not a factor.

But is second order complementation necessary for second order false beliefs (Hypothesis 3)? Our second order false belief task followed the design of other such tasks in the literature (Perner and Wimmer 1985; Sullivan et al. 1994) but we took care to distinguish the possible answers to zero order, first order and second order beliefs (Fig. 2).

\section{6) Second Order False Belief scenario}

\section{Bake Sale}

Picture 1: Sam and Maria are playing together and look out the window and see that the church is having a bake sale

Picture 2: Sam watches Maria grab her money and run out of the house and over to the church. "Ah ha," Sam says. "She's going to buy chocolate chip cookies, her favorite." And then Sam goes back to playing with his toys 
Picture 3: On her way to the bake sale, Maria runs into the mailman and tells him, "I'm going to get a nice pumpkin pie for Grandma."

Probe: Does Sam know that Maria is going to get a pumpkin pie?

Picture 4: When Maria gets to the bake sale, she finds out that all they have are brownies. So she couldn't buy a pumpkin pie for her grandma, but buys a bunch of brownies to bring back to her family instead

Probe: Does Sam know that she bought brownies?

1st order FB: What did Maria think (she) was (going to buy) at the bake sale?

Picture 5: Back at the house, Mom comes in and says to Sam "I noticed that the church is having a bake sale." "Oh yes," Sam says. "Maria went there." Then Mom asks, "oh, what does Maria think they're selling at the bake sale?"

\section{2nd order FB: What does Sam tell his mom? Why does he tell her that?}

What is required to answer correctly? The stories do not use second-order complements, so the task does not entail processing second order language. The child must instead make the connection across the discourse in order to answer. The steps are something like this:

17) "Ah ha," Sam says. "She's going to buy chocolate chip cookies, her favorite." $\rightarrow$ Sam thinks Maria wants to buy chocolate chip cookies

$\rightarrow$ Sam thinks that Maria thinks they are selling chocolate chip cookies

$\rightarrow$ What does Sam tell his Mom?

In other words, they must represent the second order belief:

Sam thinks [that Maria thinks [they are selling $x$ ]]

Hollebrandse et al. (2008) tested eighteen 6-year-old children on the following tasks:

\section{i. Second order False Belief (4 stories)}

e.g. What does Sam tell his Mom? (chocolate chip cookies)

ii. Second order Complements (6 items)

e.g. What did Billy tell Jane? (His Mom said that comic books are stupid)

Notice in Figs. 3 and 4 that the children passed the first order false belief question within the second order task at $91 \%$. Since these same children mostly failed the second order "attitudinal" complements, understanding second order complements does not seem to be a prerequisite for first order FB understanding. In addition, we did not find that single complementation leads immediately to recursive complementation. There is at least a year or two between mastery of single complements with false propositions, and our second order "attitudinal" complements task. Second, and highly surprisingly, we found that the 6-year-old children were significantly better at 2nd order False Belief than at our 2nd order attitudinal complementation task. This is contradictory to Hypothesis 3 that recursive complementation is needed to represent second order false beliefs. 


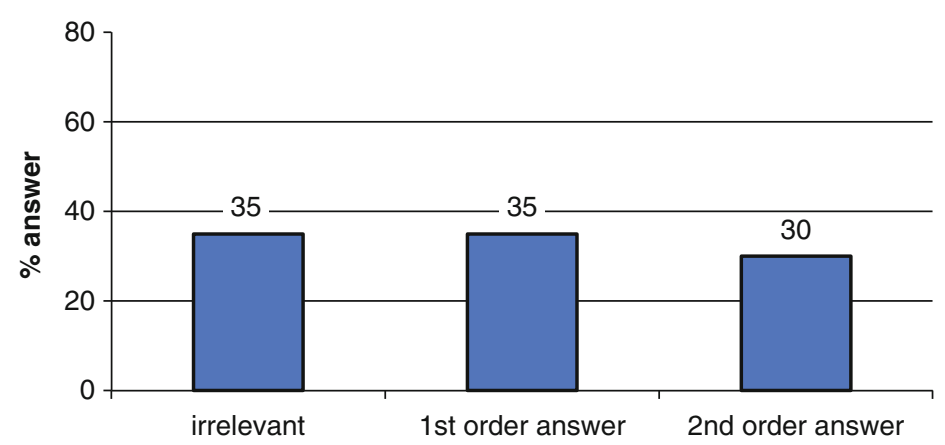

Fig. 3 Data from Hollebrandse et al. (2008) on understanding second order complements with attitudes (Figure: author's own)

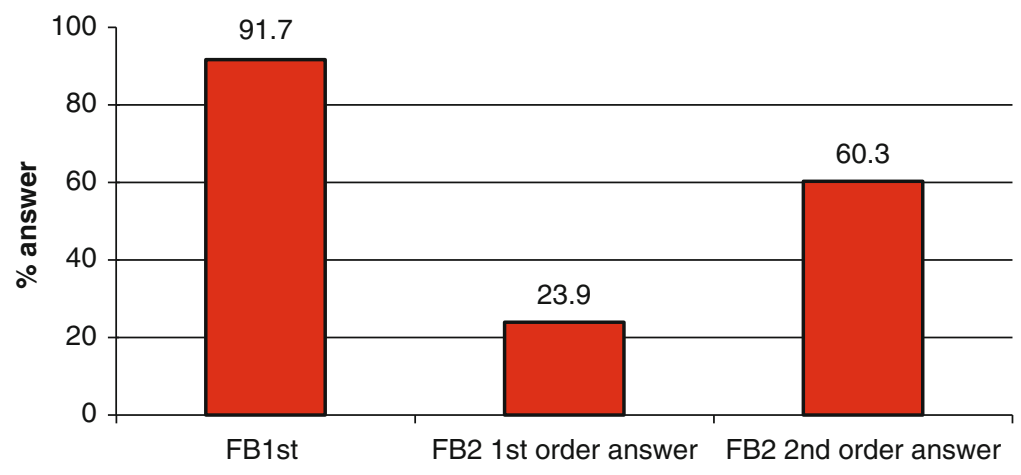

Fig. 4 Data from Hollebrandse et al. (2008) on understanding first and second order false beliefs (Figure: author's own)

Why is the second order attitudinal complementation task so hard? Some adults question our second order complementation task. Billy did "utter the words" that "comic books are stupid". Is this ambiguity the cause of the children's errors? A related explanation considers factivity: though say is not generally factive, a verb like know reveals the impact of factivity in the second verb:

18) Billy told Jane that Mom said that comic books are stupid

19) Billy told Jane that Mom knows that comic books are stupid

In 18), the factivity of the lower clause is inherited by the embedding clause, in that Billy could only say that Mom knows $p$ if Billy also knew $p$. i.e. that Billy also knows that comic books are stupid. Suppose children (and some adults) consider "say" to have such inherited factive properties? In that case they might answer that Billy said that comic books are stupid, and our attempt to manipulate 
attitude differences was ineffective. This raises a further possibility that ties in with Hypothesis 4 and brings recursion back into the picture:

In order to recognize genuine complement recursion, children need truth-value contrasts between clauses.

Suppose it is the case that the sentences we constructed for second order complementation were not sufficiently marked as recursive because the truth value was not contrastive.

20) Billy told Jane [that Mom said [that comic books are stupid]].

The second complement could be argued not to have a truth value: it is Mom's evaluation, and we could merely disagree. Just as in the first order case, where false complements permit false belief reasoning, perhaps genuine second-order complements that mark mistakes/changes in truth value are the real prerequisite for second-order belief reasoning.

21) Billy told Jane [that Mom said [that it was raining]]. (It's not.)

There is some evidence that evaluative verbs are (sometimes) not recursive. The English verb "to consider" is not recursive:

22) Billy considered Jane to consider comic books stupid

and the Dutch verb vinden (another evaluative) is non-recursive (Hollebrandse and Roeper 2014, this volume). Perhaps only truth contrasts guarantee recursivity of particular verbs. We need to test the relationship between second order complements that mark truth-value changes, and second order false belief tasks.

\section{Some Preliminary Evidence}

The hypothesis is being subject to test on a large scale, serendipitously. As part of an intervention study exploring the effectiveness of an integrated preschool curriculum with a large sample of low-income children in Texas and Florida (de Villiers et al. 2009), we designed language and theory of mind tests to be repeated at intervals throughout the age range 2.5-7 years. As a consequence of the need to test the older children, we developed extended false belief measures, using second order belief stories, and we also expanded on our complement comprehension measures so that they too were second order. This will allow us to test whether second order complements that entail truth-value changes predict second order false belief reasoning. However, at the moment we only have preliminary data as the longitudinal study is not completely analyzed. 


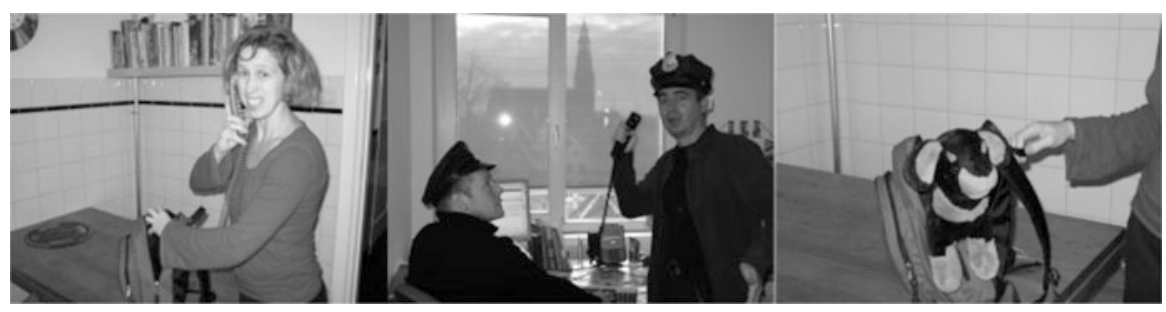

Fig. 5 A sample series of pictures from the new second order complement comprehension task, contrasting truth value (Figure: author's own)

At the time of writing we have data on 191 five- to six-year-olds in low-income preschools and daycares in Florida and Texas, being tested as part of a large NIHfunded study on curriculum intervention for school readiness. The children were assessed three times during the critical year of intervention, and then once a year later at follow-up. Ultimately we will have another year of follow-up data. These data are from the first follow-up testing, 1 year out of the program.

Here we only provide a brief report of the results of testing the children as the full results are still being compiled. In a large battery of cognitive, emotional and literacy/language assessments, the children all received the following tests relevant to the present questions:

a) 1st order complements (de Villiers and Pyers 2002)

b) 1st order false belief (a nonverbal analog of the standard task, see Schick et al. 2007)

c) 2nd order false belief (Hollebrandse et al. 2008)

d) 2nd order complements (contrasting truth (Hollebrandse and Van Hout 2009)

The latter task was devised to extend the single false complement task to second order, and goes as follows. Three pictures (Fig. 5) are shown to illustrate a story (23), and then a wh-question (24) is asked to diagnose the complements as before:

23) The woman called the policeman and said that there was a rat in her backpack.

The policeman did not hear her very well and said,

"What? The woman said there's $a$ cat in her backpack! No, it must be $a$ scarf."

But look. It's just a stuffed toy.

24) What did the policeman say the woman said t was in her backpack?

First, consider the result in Fig. 6, demonstrating that the children have mastered first order complementation as indexed by the standard wh-question.

We checked whether we obtained the usual contingency between passing first order complementation and the nonverbal first order false belief, and the results 


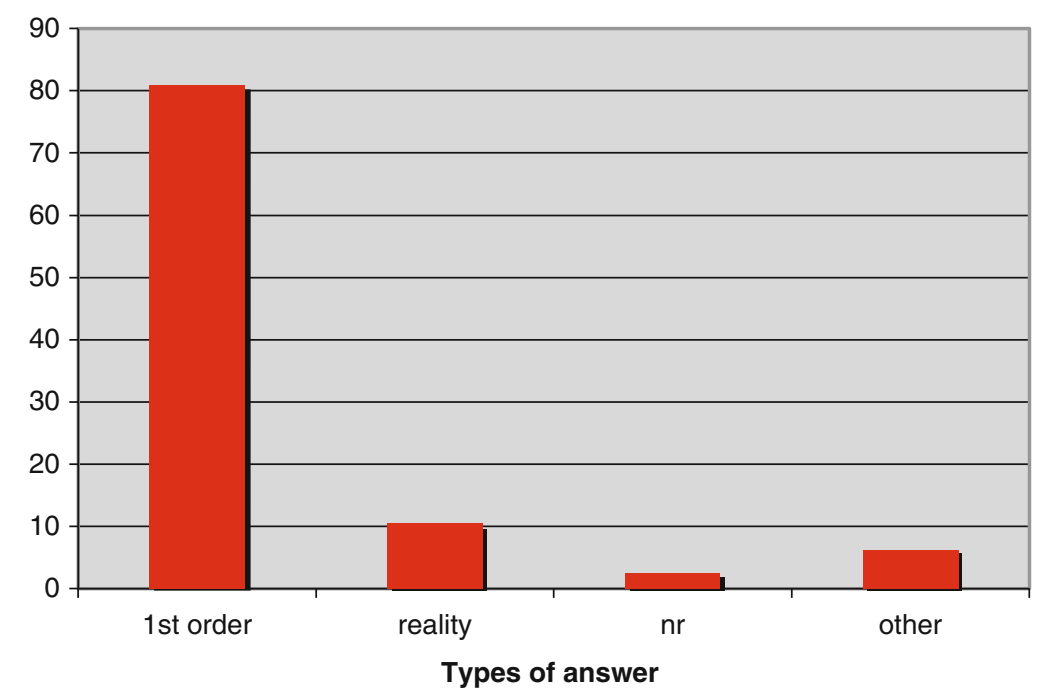

Fig. 6 Evidence of passing first order belief in the longitudinal study (Figure: author's own)

Table 1 First order complementation and nonverbal first order false belief

\begin{tabular}{|l|l|r|r|r|r|r|r|}
\hline \multicolumn{2}{|c|}{ Contingency } & \multicolumn{6}{|c|}{ Number of complements correct } \\
\cline { 3 - 8 } & & 0 & 1 & \multicolumn{1}{c|}{2} & \multicolumn{1}{c|}{3} & \multicolumn{1}{c|}{ Total } \\
\hline Number of & 0 & 5 & 8 & 7 & 26 & 36 & 82 \\
nonverbal & 1 & 1 & 4 & 9 & 10 & 38 & 62 \\
false beliefs & 2 & 1 & 1 & 1 & 6 & 24 & 33 \\
correct & 2 & 0 & 0 & 1 & 1 & 12 & 14 \\
& 3 & 7 & 13 & 18 & 43 & 110 & 191 \\
\hline
\end{tabular}

are shown in Table 1. Passing the nonverbal false belief tasks is contingent on passing the complementation task, confirming previous results. As usual, we find an asymmetry: some children can pass first order complements but still not pass the first order false belief tasks.

What do the children do with the scenarios of second order complementation? The question was for example:

24) What did the policeman say the woman said was in her backpack?

The results so far indicate: 
Earlier study 6 year olds

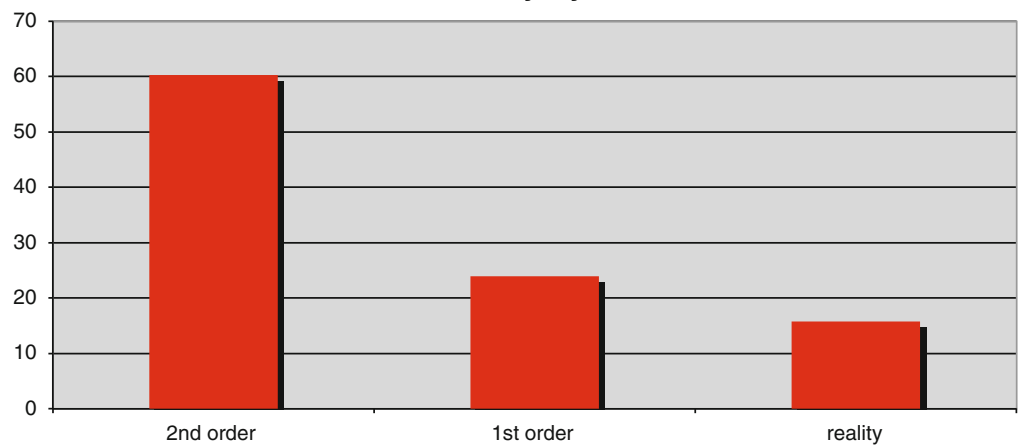

New Study 5-6 year olds

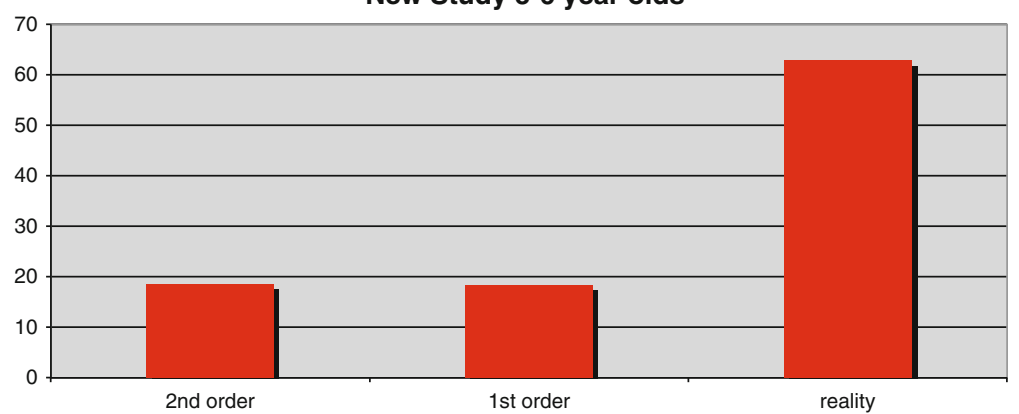

Fig. 7 Results on second order false beliefs comparing two different populations (Figure: author's own)

$29 \%$ correct 2 nd order $=$ cat

$26 \% 1$ st order policeman $=$ scarf

$16 \% 1$ st order woman $=$ rat

$15 \%$ reality $=$ stuffed toy

The rate of second order answers, $29 \%$, is still outweighed by a preponderance of first order answers (42 \%). Nevertheless, there is a hint that the subjects are doing better on these second order complements than our earlier subjects did with the attitudinal complements. This larger group can be claimed to be at a less advanced stage of development than the sample of children tested previously; the largescale study is composed of low-income children from much more impoverished educational backgrounds than children in our previous work (e.g. in Hollebrandse et al. 2008). The evidence comes from comparing the two participant groups on their performance on second order false beliefs in Fig. 7: clearly this second, larger, group is not as advanced as the previous groups of children in their theory of mind reasoning. 
Given that the present subjects are less advanced in development, then it is surprising that their performance on the second order complements is at least as good as the earlier group. The possibility must be raised that having truth-varying complements may improve performance relative to evaluative complements, but a new study in which both the stories and the participants are more closely matched would be necessary to test it.

The second question was whether the new group's performance on second order, truth varying complements was better than, or preceded, their performance on second order false beliefs. Comparing Figs. 6 and 7, three comparisons make this point. We make the reasonable posit that on the second order task, second order answers are better than first order answers, and also that first order answers are more advanced than reality answers, even though they are wrong.

First, the percentage of success, that is, second order answers, is higher on the complements task than on the false belief task. Second, the number of first order answers is higher on the complement task than on the false belief task. And third, the number of reality answers is lower on the complements task than on the false belief task.

We suggest that mastery of recursive complements precedes mastery of recursive false beliefs, but are they prerequisite? It is clear that understanding second order truth-contrastive complements comes in before second order false belief reasoning. However there are as yet too few passers of second order false belief to be able to test Hypothesis 2 that recursive complements are prerequisite, as we would like to claim.

Why are second order complements needed for second order false belief reasoning? Remember that the stories do not use second-order complements: they are covert. The child must make the connection across the discourse in order to answer:

25) What does Maria think they are selling at the bake sale?

26) What does Sam tell his Mom?

We contend that the child must mentally represent the second order belief:

\section{7) Sam thinks [that Maria thinks [they are selling chocolate chip cookies]]}

Children at 5 or 6 cannot yet do this. Second-order complements are not yet securely represented (even with communication verbs) even when they are overt. Until they can handle them, indexed by our comprehension test, children will fail second-order false belief tests.

In another year, we will be able to trace whether there is a genuine contingency between passing second-order truth-contrastive complements and succeeding on second-order belief reasoning. All we can say at present is that success on complements is "leading the way". 


\section{Conclusion}

It is not recursion of complements per se that allows children to use them for representing first order false beliefs. Hypothesis 4 finds support:

Hypothesis 4: In order to recognize genuine complement recursion, children need truth-value contrasts between clauses.

With that acknowledged, we may find evidence for Hypothesis 3:

Hypothesis 3: Recursive complements (i.e. truth value varying) are necessary for recursive (level $2+$ ) false belief reasoning.

In both complementation and false belief reasoning, children first treat 2-level embedding as 1-level of structure. It is as if one piece of the hierarchy is flattened, or skipped over in parsing. Single level embedding, either of complements or of propositional attitudes, does not lead instantly to second level, or recursive, embedding.

We suspect that truth contrasts provide the key at two stages. Truth contrasts provide the key that certain clauses are complements rather than adjuncts, allowing the right structure to be constructed for first order complements under certain classes of verbs. We have speculated that truth contrasts may also enable children to recognize recursion of complementation more easily compared to propositions that vary in evaluative attitude. Are truth contrasts between clauses then critical triggers of sentence recursion? If this is right, then:

Truth contrasts -> sentence recursion -> false belief recursion.

This would occur at two levels, perhaps one at age 4 years and a second at age 6 years. Miller (2009), in a review of empirical work on second order theory of mind in children, calls for more research in this domain. In particular, he argues that the theories that explain the onset of first order theory of mind are often not easily adapted for explaining second order theory of mind. The story presented here is also incomplete and there remains much more to be explored.

\section{References}

Apperly, I. (2011). Mindreaders: The cognitive basis of "Theory of Mind". Hove: Psychology Press.

Baillargeon, R., Scott, R., \& He, Z. (2010). False belief understanding in infants. Trends in Cognitive Science, 14, 110-118.

Bartsch, K., \& Wellman, H. M. (1995). Children talk about the mind. New York: Oxford University Press.

Carruthers, P. (2002). The cognitive functions of language. Behavioral and Brain Sciences, 25, 657-719.

Chomsky, N. (2005). On phases. Unpublished paper, MIT. Pdf available online at http://dmtr.ru/ blog/omniling/ 
Chomsky, N. (2010). Some simple evo-devo theses: How true might they be for language? In R. K. Larson, V. Deprez, \& H. Yamakido (Eds.), The evolution of human language. Cambridge: Cambridge University Press.

Collins, J. (2000). Theory of Mind, logical form and eliminativism. Philosophical Psychology, 13, 465-490.

de Villiers, J. G. (1995, March). Steps in the mastery of sentence complements. Paper presented at the meeting of the Society for Research in Child Development, Indianapolis, Indiana.

de Villiers, J. G. (1999). On acquiring the structural representations for false complements. In B. Hollebrandse (Ed.), New perspectives on language acquisition. Amherst: University of Massachusetts Press.

de Villiers, J. G. (2005a). Can language acquisition give children a Point of View? In J. W. Astington \& J. A. Baird (Eds.), Why language matters for Theory of Mind. Oxford: Oxford University Press.

de Villiers, P. A. (2005b). The role of language in Theory-of-Mind development: What deaf children tell us. In J. W. Astington \& J. A. Baird (Eds.), Why language matters for Theory of Mind. Oxford: Oxford University Press.

de Villiers, J. G. (2009, September). On building up a sufficient representation for belief: Tense, point of view and Wh-movement. Plenary address presented at the meeting of Generative Approaches to Language Acquisition, Lisbon, Portugal.

de Villiers, J. G., \& de Villiers, P. A. (2003). Language for thought: Coming to understand false beliefs. In D. Gentner \& S. Goldin-Meadow (Eds.), Language in mind: Advances in the study of language and thought. Cambridge, MA: MIT Press.

de Villiers, J. G., \& Pyers, J. (2002). Complements to cognition: A longitudinal study of the relationship between complex syntax and false-belief understanding. Cognitive Development, 17, 1037-1060.

de Villiers, J. G., Roeper, T., \& Vainikka, A. (1990). The acquisition of long distance rules. In L. Frazier \& J. G. de Villiers (Eds.), Language processing and acquisition. Dordrecht: Kluwer.

de Villiers, P. A., Burns, F., \& Pearson, B. Z. (2003). The role of language in the Theory of Mind development of language-impaired children: Complementing theories. In B. Beachley, A. Brown, \& F. Conlin (Eds.), Proceedings of the 27th annual Boston University conference on language development. Somerville: Cascadilla Press.

de Villiers, J. G., de Villiers, P. A., Hobbs, K., Taylor, H., \& Swank, P. (2009, April). Language and Theory of Mind in a large low-income sample of preschoolers studied longitudinally. Paper presented at the meeting of the Society for Research in Child Development, Denver, CO.

de Villiers, J. G., de Villiers, P. A., \& Roeper, T. (2011). Wh-questions: Moving beyond the first phase. Lingua, 121, 352-366.

de Villiers, J., Roeper, T., Harrington, E., \& Gadilauskas, E. (2012). Tense and truth in children's question answering. In A. K. Biller, E. Y. Chung, \& A. E. Kimball (Eds.), Proceedings of the 36th annual Boston University conference on language development. Somerville: Cascadilla Press.

Dennett, D. C. (1987). The intentional stance. Cambridge, MA: MIT Press.

Dienes, Z., \& Perner, J. (2002). What sort of representation is conscious? Behavioral and Brain Sciences, 25, 336-338.

Diessel, H., \& Tomasello, M. (2001). The acquisition of finite complement clauses in English: A corpus-based analysis. Cognitive Linguistics, 12-2, 97-141.

Doherty, M. J. (2006). The development of mentalistic gaze understanding. Infant and Child Development, 15, 179-186.

Everett, D. (2008). Don't sleep, there are snakes: Life and language in the Amazonian jungle. New York: Pantheon Books.

Fodor, J. (1975). The language of thought. New York: Harvester Press.

Fodor, J. (2008). LoT2: Language of thought revisited. Oxford: Oxford University Press.

Hale, C. M., \& Tager-Flusberg, H. (2003). The influence of language on Theory of Mind: A training study. Developmental Science, 6, 346-359. 
Harris, P. L. (2005). Conversation, pretence, and Theory of Mind. In J. W. Astington \& J. Baird (Eds.), Why language matters for Theory of Mind. New York: Oxford University Press.

Hauser, M., Chomsky, N., \& Fitch, W. T. (2002). The language faculty: What is it, who has it, and how did it evolve? Science, 298, 1569-1579.

Hobbs, K., de Villiers, P., de Villiers, J., \& Peña, E. (in preparation). Evidence against a bilingual advantage in false belief reasoning.

Hollebrandse, B., \& Roeper, T. (2007, April). Recursion and propositional exclusivity. Paper presented at the Recursion in Human Languages conference, Normal, IL.

Hollebrandse, B., \& Roeper, T. (2014). Empirical results and formal approaches to recursion in acquisition. In T. Roeper \& M. Speas (Eds.), Recursion: Complexity in cognition. Dordrecht: Springer.

Hollebrandse, B., \& Van Hout, A. (2009, February 7). What did who say about who? The acquisition of double embedding. Taalkunde in Nederland dag.

Hollebrandse, B., Hobbs, K., de Villiers, J. G. \& Roeper, T. (2006, December). Second order things. Paper presented at UMass-UConn-Smith Language Acquisition Workshop, Amherst, MA.

Hollebrandse, B., Hobbs, K., de Villiers, J. G., \& Roeper, T. (2008). Second order embedding and second order false belief. In A. Gavarró \& M. J. Freitas (Eds.), Language acquisition and development: Proceedings of GALA 2007 (pp. 270-280). Cambridge: Cambridge Scholar Press.

Hutto, D. (2008). Folk psychological narratives. Cambridge, MA: MIT press (Bradford books).

Jackendoff, R. (2011). What is the human language faculty? Two views. Language, 87, 586-624.

Jacques, S., \& Zelazo, P. D. (2005). Language and the development of cognitive flexibility: Implications for Theory of Mind. In J. W. Astington \& J. A. Baird (Eds.), Why language matters for Theory of Mind (pp. 144-162). New York: Oxford University Press.

Loewenstein, J., \& Gentner, D. (2005). Relational language and the development of relational mapping. Cognitive Psychology, 50, 315-353.

Lohmann, H., \& Tomasello, M. (2003). The role of language in the development of false belief understanding: A training study. Child Development, 74, 1130-1144.

Low, J. (2010). Preschoolers' implicit and explicit false-belief understanding: Relations with complex syntactical mastery. Child Development, 81, 597-615.

Miller, S. A. (2009). Children's understanding of second-order mental states. Psychological Bulletin, 135(5), 749-773.

Milligan, K., Astington, J., \& Dack, L. A. (2007). Language and Theory of Mind: Meta-analysis of the relation between language ability and false-belief understanding. Child Development, 78 , 622-646.

Nelson, K. (2005). Language pathways into the community of minds. In J. W. Astington \& J. A. Baird (Eds.), Why language matters for Theory of Mind. Oxford: Oxford University Press.

Onishi, K. H., \& Baillargeon, R. (2005). Do 15-month-old infants understand false beliefs? Science, 308, 255-258.

Perner, J. (2010). Who took the cog out of cognitive science? Mentalism in an era of anticognitivism. In P. A. Frensch \& R. Schwarzer (Eds.), Cognition and neuropsychology: International perspectives on psychological science (Vol. 1, pp. 241-261). London: Psychology Press.

Perner, J., \& Ruffman, T. (2005). Infants' insight into the mind: How deep? Science, 308, 214-216.

Perner, J., \& Wimmer, H. (1985). John thinks that Mary thinks that: Attribution of second-order beliefs by 5- to 10-year-old children. Journal of Experimental Child Psychology, 39, 437-471.

Pyers, J., \& Senghas, A. (2009). Language promotes false-belief understanding: Evidence from learners of a new sign language. Psychological Science, 20, 805-812.

Roeper, T. (2007). The prism of grammar. Cambridge: MIT Press.

Ruffman, T., Slade, L., Rowlandson, K., Rumsey, K., \& Garnham, A. (2003). How language relates to belief, desire, and emotion understanding. Cognitive Development, 18, 139-158.

Sacks, O. (1985). The man who mistook his wife for a hat, and other clinical tales. New York: Summit Books. 
Sauerland, U., \& Trotzke, A. (2011). Biolinguistic perspectives on recursion: Introduction [special issue]. In U. Sauerland \& A. Trotzke (Eds.), Biolinguistics 5(1-2), 1-9. http://www. biolinguistics.eu

Schick, B., de Villiers, P. A., de Villiers, J. G., \& Hoffmeister, R. (2007). Language and Theory of Mind: A study of deaf children. Child Development, 78, 376-396.

Segal, G. (1998). Representing representations. In P. Carruthers \& J. Boucher (Eds.), Language and thought. Cambridge: Cambridge University Press.

Southgate, V., Senju, A., \& Csibra, G. (2007). Action anticipation though attribution of false belief by 2-year-olds. Psychological Science, 18, 587-592.

Sullivan, K., Zaitchik, D., \& Tager-Flusberg, H. (1994). Preschoolers can attribute second-order beliefs. Developmental Psychology, 30, 395-402.

Tager-Flusberg, H., \& Joseph, R. (2005). How language facilitates the acquisition of false belief in children with autism. In J. W. Astington \& J. A. Baird (Eds.), Why language matters for Theory of Mind. Oxford: Oxford University Press.

Wierzbicka, A. (1996). Semantics: Primes and universals. Oxford: Oxford University Press.

Woolfe, T., Want, T., \& Siegal, T. (2002). Signposts to development: Theory of Mind in deaf children. Child Development, 73, 768-778. 